\title{
CARACTERIZAÇÃO TERMOFÍSICA DE POLPAS DE BACURI ${ }^{1}$
}

\author{
Marcelo B. MUNIZ², Alexandre José de M. QUEIROZ², \\ Rossana M.F. de FIGUEIRÊDO ${ }^{3, *}$, Maria Elita M. DUARTE ${ }^{3}$
}

\begin{abstract}
RESUMO
Foram estudadas as propriedades termofísicas calor específico, massa específica, difusividade térmica e condutividade térmica de polpas de bacuri em diferentes concentrações. O calor específico foi determinado pelo método do calorímetro de mistura; a massa específica foi determinada em picnômetro; a difusividade térmica foi obtida utilizando-se o método do cilindro infinito; a condutividade térmica foi determinada a partir dos resultados experimentais obtidos para massa específica, calor específico e difusividade térmica. Todas as propriedades também foram estimadas teoricamente com o uso de equações propostas em literatura e por meio de regressões dependentes da concentração. Entre os resultados experimentais o calor específico situou-se entre 3,616 a $2,986 \mathrm{~kJ} / \mathrm{kg}^{\circ} \mathrm{C}$; a massa específica variou de $1.008,40$ a $1.048,36 \mathrm{~kg} / \mathrm{m}^{3}$; a difusividade térmica variou de $1,637 \times 10^{-7}$ a $1,787 \times 10^{-7}$ $\mathrm{m}^{2} / \mathrm{s}$; a condutividade térmica variou de 0,50 a $0,64 \mathrm{~W} / \mathrm{m}^{\circ} \mathrm{C}$. Exceto a massa específica, o valor de todas as propriedades diminuiu com o aumento da concentração. A condutividade térmica foi melhor representada por equação do tipo exponencial. As demais propriedades foram bem ajustadas por equações do tipo quadrática.

Palavras-chave: polpa de bacuri, concentração, propriedades termofísicas.
\end{abstract}

\section{SUMMARY}

THERMOPHYSICAL CHARACTERIZATION OF BACURI PULP. Thermophysical properties, specific heat, density, thermal diffusivity and thermal conductivity of bacuri pulps at different concentrations were studied. The specific heat was determined by the mixture calorimeter method; the density was determined by the pycnometer; the thermal diffusivity was obtained by using the infinite cylinder method; the thermal conductivity was determined from the experimental results obtained for density, specific heat and thermal diffusivity. All the properties were also theoretically determined using proposals equations and regressions. Among the experimental results the specific heat varied from 3.616 to $2.986 \mathrm{~kJ} / \mathrm{kg}^{\circ} \mathrm{C}$; the density varied from $1,008.40$ to $1,048.36 \mathrm{~kg} / \mathrm{m}^{3}$; the thermal diffusivity varied between $1.637 \times 10^{-7}$ and $1.787 \times 10^{-7} \mathrm{~m}^{2} / \mathrm{s}$; the thermal conductivity varied from 0.50 to $0.64 \mathrm{~W} / \mathrm{m}^{\circ} \mathrm{C}$. Except for the density, the value of all the properties decreased, whereas the concentration increased. The thermal conductivity was fitted better by exponential equation. The other properties were well fitted by quadratic equations.

Keywords: bacuri pulp, concentration, thermophysical properties.

\section{1 - INTRODUÇÃO}

A produção mundial de frutas, no ano de 2004, foi de 675,1 milhões de toneladas em 71,5 milhões de hectares, e o Brasil contribuiu com 39 milhões de toneladas, conferindo-lhe a posição de terceiro maior produtor de frutas frescas do mundo, superado apenas pela China e Índia, com 161 e 58 milhões de toneladas, respectivamente [1].

O bacurizeiro (Platonia insignis Mart.), espécie frutífera da família das gutiferáceas, é uma planta arbórea, tipicamente tropical, cujas áreas de ocorrência abrangem os Estados do Pará, Maranhão, Piauí, Goiás e Mato Grosso, alcançando também o Paraguai. Os centros de origem

\footnotetext{
${ }^{1}$ Recebido para publicação em 10/5/2005. Aceito para publicação em 28/4/2006 (001522)

${ }^{1}$ Parte da dissertação do primeiro autor

${ }^{2}$ Engenheiro agrônomo

${ }^{3}$ Centro de Ciências e Tecnologia da Universidade Federal de Campina Grande (UFCG)

Avenida Aprígio Veloso, 882

Campina Grande (PB) - CEP 58109-970

E-mail: rossana@deag.ufcg.edu.br

*A quem a correspondência deve ser enviada
}

e de diversidade da espécie estão localizados no Pará, onde são encontradas ampla variação de forma e tamanho de frutos, rendimento e qualidade de polpa, além de outras características de interesse econômico [2]. Seu fruto pode ser utilizado tanto na forma in natura quanto na agroindústria [3].

As produções de bacuri são comercializadas, sobretudo em centrais de abastecimento e feiras livres de Belém (PA), São Luís (MA) e Teresina (PI) e não têm sido suficientes para atender à demanda crescente do mercado consumidor destas capitais. Na forma de polpa congelada, sua comercialização ocorre de forma especial nas grandes redes de supermercados dessas capitais, a preços superiores aos de outras frutas tropicais de valor estabelecido, como o cupuaçu, o cajá, a goiaba e a graviola.

Por não constituir ainda uma cultura comercialmente estabelecida, a produção de frutos é decorrente, na quase totalidade, de atividades extrativistas, sendo raros os pomares com a espécie [4]. Porém, apesar do seu elevado potencial econômico, muito pouco tem sido feito para o conhecimento e uso dessa espécie, quer na área de coleta, conservação, caracterização e avaliação de germoplasma, quer na de melhoramento genético, visando o desenvol- 
vimento de cultivares e de práticas específicas de cultivo e manejo [5]. Atualmente, o seu sistema de exploração é quase exclusivamente extrativista.

Inexistem, de igual forma, informações sobre as propriedades termofísicas da polpa do bacuri, importantes em sistemas industriais onde se aplicam processos de aquecimento, resfriamento e estocagem sob temperatura controlada. Nestes processos as características dos materiais entram como dados no dimensionamento de sistemas de aquecimento, resfriamento e manutenção da temperatura. Os dados característicos de cada material também permitem prever as relações tempo-temperatura necessárias para atingir a temperatura desejada no processo, seja de aquecimento ou de resfriamento.

Este trabalho foi realizado com o objetivo de se estudar as propriedades termofísicas calor específico, massa específica, condutividade térmica e difusividade térmica de polpa de bacuri em concentrações de 5; 7,5; 10; 12,5; $15 ; 17,5$ e $20^{\circ}$ Brix.

\section{2 - MATERIAL E MÉTODOS}

\section{1 - Matéria-prima}

A polpa do bacuri foi oriunda da zona rural circunvizinha da cidade de São Luís (MA), cujo período de colheita ocorre entre dezembro e março. Foi obtida por despolpamento manual de frutos maduros, embalada em sacos de polietileno, congelada e transportada em caixas térmicas até as instalações do Laboratório de Armazenamento e Processamento de Produtos Agrícolas, Deag, da Universidade Federal de Campina Grande, em Campina Grande (PB).

A polpa de bacuri integral, com concentração de $20^{\circ}$ Brix, foi diluída com água destilada, para a obtenção das amostras nas concentrações de $5 ; 7,5 ; 10 ; 12,5 ; 15$ e $17,5^{\circ}$ Brix.

\section{2 - Propriedades termofísicas}

\subsection{1 - Calor específico}

Determinou-se o calor específico das polpas de bacuri, em sextuplicata, utilizando-se um calorímetro de mistura, que consiste em uma garrafa térmica (frasco Dewar), com capacidade para $1.000 \mathrm{~mL}$, envolvida em camada de $5 \mathrm{~cm}$ de isolante térmico (lã de vidro), inserida em uma carcaça de PVC. A garrafa era fechada com rolha de borracha, dotada de furo por onde era introduzida a haste de um termopar a fim de se acompanhar a temperatura interna.

Para se obter o calor específico das amostras colocavase, inicialmente, no calorímetro, uma massa de água $\left(\mathrm{m}_{1}\right)$ à temperatura ambiente $\left(\mathrm{T}_{1}\right)$. Em seguida, era adicionada uma massa de água resfriada $\left(\mathrm{m}_{2}\right)$ em temperatura $\mathrm{T}_{2}$; agitava-se o calorímetro e se registrava a massa $\mathrm{m}_{3}$ e a temperatura $\mathrm{T}_{3}$ no equilíbrio; depois, acrescentava-se uma massa de amostra $\left(\mathrm{m}_{\mathrm{p}}\right)$ em temperatura $\left(\mathrm{T}_{4}\right)$; agitava-se o equipamento e se registrava a temperatura no equilíbrio $\left(\mathrm{T}_{5}\right)$.
Para determinar a capacidade calorífica do calorímetro, utilizou-se o balanço de calor da Equação 1 descrito por SILVA et al. [6].

$\mathrm{C}_{1} \mathrm{~m}_{1}\left(\mathrm{~T}_{1}-\mathrm{T}_{3}\right)+\mathrm{C}_{\text {cal }}\left(\mathrm{T}_{1}-\mathrm{T}_{3}\right)=\mathrm{C}_{2} \mathrm{~m}_{2}\left(\mathrm{~T}_{3}-\mathrm{T}_{2}\right)$

Onde:

$\mathrm{C}_{1} \mathrm{e}_{2}$ - Calor específico da água $\left(\mathrm{cal} / \mathrm{g}^{\circ} \mathrm{C}\right)$

$\mathrm{m}_{1}$ - Massa de água à temperatura ambiente (g)

$\mathrm{m}_{2}$ - Massa de água refrigerada (g)

$\mathrm{C}_{\text {cal }}$ - Capacidade calorífica do calorímetro (cal/ ${ }^{\circ} \mathrm{C}$ )

Para determinação do calor específico do produto, foi usada a Equação 2 descrita por SILVA et al. [6]:

$\mathrm{m}_{\mathrm{p}} \mathrm{C}_{\mathrm{p}}\left(\mathrm{T}_{4}-\mathrm{T}_{5}\right)=\mathrm{C}_{1} \mathrm{~m}_{3}\left(\mathrm{~T}_{5}-\mathrm{T}_{3}\right)+\mathrm{C}_{\text {cal }}\left(\mathrm{T}_{5}-\mathrm{T}_{3}\right)$

Sendo:

$\mathrm{m}_{\mathrm{p}}$ - Massa do produto $(\mathrm{g})$

$\mathrm{C}_{\mathrm{p}}-$ Calor específico do produto $\left(\mathrm{cal} / \mathrm{g}^{\circ} \mathrm{C}\right)$

$\mathrm{C}_{1}$ - Calor específico da água $\left(\mathrm{cal} / \mathrm{g}^{\circ} \mathrm{C}\right)$

$\mathrm{m}_{3}$ - Soma das massas $\mathrm{m}_{1}$ e $\mathrm{m}_{2}$

$\mathrm{T}_{4}$ - Temperatura inicial do produto $\left({ }^{\circ} \mathrm{C}\right)$

$\mathrm{T}_{5}-$ Temperatura de equilíbrio $\left({ }^{\circ} \mathrm{C}\right)$

Para determinação do calor específico teórico foram utilizadas as equações apresentadas na Tabela 1, as quais foram escolhidas em razão de estimarem a massa específica em função da concentração ou quantidade de água e terem sido propostas para alimentos e sucos de frutas (laranja e tamarindo).

TABELA 1 - Equações para o cálculo do calor específico de alimentos

\begin{tabular}{lc}
\hline Equação & Referência \\
\hline$C_{p}=1,675+2,512 X_{w}$ & {$[7]$} \\
$C_{p}=0,837+3,349 X$ & {$[8]$} \\
$C_{p}=1,465+2,721 X_{w}$ & {$[8]$} \\
$C_{p}=4,1713-0,0279 \mathrm{~B}$ & {$[9]$} \\
$C_{p}=4,18+\left(6,839 \times 10^{-5} \mathrm{~T}-5,03 \times 10^{-2}\right) \mathrm{S}$ & \\
\hline$C_{p}-$ Calor específico $\left(\mathrm{kJ} / \mathrm{kg}^{\circ} \mathrm{C}\right)$ & \\
$\mathrm{X}_{\mathrm{w}}-$ fração mássica da água (adimensional) \\
$\mathrm{B}-$ Concentração $\left({ }^{\circ} \mathrm{Brix}\right)$ \\
$\mathrm{S}-$ Sólidos totais $(\%)$ \\
$\mathrm{T}-$ Temperatura ( $\left.{ }^{\circ} \mathrm{C}\right)$
\end{tabular}




\subsection{2 - Massa específica}

Para a determinação das massas específicas das polpas de bacuri nas concentrações de 5,0; 7,5; 10,0; 12,5; 15,0; 17,5 e $20,0^{\circ} \mathrm{Brix}$, em sextuplicata, utilizou-se picnômetro de $25 \mathrm{~mL}$, previamente calibrado com água destilada, nas temperaturas de 25 e $30^{\circ} \mathrm{C}$. O cálculo da massa específica foi realizado através da Equação 3, que estabelece relação entre massa e volume [11].

$\rho=\frac{m}{v}$

Na qual :

$\rho$ - Massa específica do produto $\left(\mathrm{kg} / \mathrm{m}^{3}\right)$

$\mathrm{v}$ - Volume do picnômetro $\left(\mathrm{m}^{3}\right)$

$\mathrm{m}$ - Massa do produto $(\mathrm{kg})$

Para a determinação da massa específica teórica, utilizaram-se equações existentes na literatura, apresentadas na Tabela 2 , as quais foram selecionadas em razão de serem, principalmente, função da temperatura e em alguns casos da concentração ou umidade e também de uma delas ter sido proposta para sucos em geral (referência [12]) e as outras para polpas de frutas típicas da Amazônia (referências [13] e [14]).

TABELA 2 - Equações para cálculo da massa específica em alimentos

\begin{tabular}{lc}
\hline Equação & Referência \\
\hline$\rho=1002+4,61 B-0,460 T+7,001 \times 10^{-3} T^{2}-9,175 \times 10^{-5} T^{3}$ & [12] \\
$\rho=1.035,3-0,07429 T-0,006437 T^{2}$ & [13] \\
$\rho=1.068,65-0,4579 X_{\mathrm{w}}-0,3867 T$ & \\
\hline$\rho-$ Massa específica $\left(\mathrm{kg} / \mathrm{m}^{3}\right)$ & \\
$\mathrm{X}_{\mathrm{w}}-$ Umidade $(\%)$ & \\
$\mathrm{B}-$ Concentração $\left({ }^{\circ} \mathrm{Brix}\right)$ & \\
$\mathrm{T}-$ Temperatura $\left({ }^{\circ} \mathrm{C}\right)$ &
\end{tabular}

\subsection{3 - Difusividade térmica}

Para determinação experimental da difusividade térmica, em triplicata, utilizou-se a metodologia proposta por DICKERSON [15]. O aparato utilizado consistiu em um cilindro metálico medindo 0,049 m de diâmetro interno, 0,23 $\mathrm{m}$ de comprimento e vedado nas extremidades por meio de rolhas de nylon. O cilindro foi provido de dois termopares, um soldado à superfície externa e o outro inserido através do centro de uma das rolhas, de modo a aferir a temperatura no centro radial e em posição eqüidistante das extremidades.
O cilindro era preenchido com as amostras, adicionadas de $1 \%$ de gelatinizante para evitar a convecção natural, e o aparato era imerso em banho termostático, ajustado para aquecimento até a temperatura de $60^{\circ} \mathrm{C}$. As temperaturas na superfície e no centro do cilindro eram registradas em intervalos de $1 \mathrm{~min}$.

Para os cálculos de difusividade térmica utilizou-se a Equação 4 [15].

$\alpha=\frac{A R_{\mathrm{c}}^{2}}{4\left(T_{s}-T_{c}\right)}$

Então:

$\alpha$ - Difusividade térmica $\left(\mathrm{m}^{2} / \mathrm{s}\right)$

A - Taxa de aquecimento do banho $\left({ }^{\circ} \mathrm{C} / \mathrm{min}\right)$

$\mathrm{R}_{\mathrm{c}}$ - Raio do cilindro (m)

$\mathrm{T}_{\mathrm{s}}$ - Temperatura na superfície do cilindro de raio $\mathrm{R}\left({ }^{\circ} \mathrm{C}\right)$

$\mathrm{T}_{\mathrm{c}}-$ Temperatura no centro do cilindro $\left({ }^{\circ} \mathrm{C}\right)$

A difusividade térmica das amostras também foi estimada por meio de equações teóricas, apresentadas na Tabela 3, as quais foram escolhidas por estimarem a difusividade térmica em função da temperatura e terem sido propostas para alimentos e sucos (laranja e tomate).

TABELA 3 - Equações utilizadas para estimativa teórica da difusividade térmica

\begin{tabular}{|c|c|}
\hline Equação & Referência \\
\hline$\alpha=\left[0,057363 X_{w}+0,000288 \cdot T\right] 10^{-6}$ & {$[16]$} \\
\hline$\alpha_{\exp }=7,683 \times 10^{-8}+5,9839 \times 10^{-8} X_{w}+0,02510 \times 10^{-8} \mathrm{~T}$ & {$[17]$} \\
\hline $\begin{array}{l}\alpha=\alpha_{w} X_{w}+\alpha_{s} X_{s} \\
\text { em que: }\end{array}$ & {$[18]$} \\
\hline$\alpha_{w}=\left(0,1363+0,00048754 T-0,0000016316 T^{2}\right) 10^{-6}$ & \\
\hline$\alpha_{s}=\left(0,0095075+0,0002347 T+0,0000017608 T^{2}\right) 10^{-6}$ & \\
\hline \multicolumn{2}{|l|}{$\alpha$ - Difusividade térmica $\left(\mathrm{m}^{2} / \mathrm{s}\right)$} \\
\hline \multicolumn{2}{|l|}{$\mathrm{T}$ - Temperatura $\left(\mathrm{K} ;{ }^{\circ} \mathrm{C}\right)$} \\
\hline \multicolumn{2}{|l|}{$\alpha_{w}-$ Difusividade térmica da água $\left(\mathrm{m}^{2} / \mathrm{s}\right)$} \\
\hline \multicolumn{2}{|l|}{$\alpha_{\mathrm{s}}-$ Difusividade térmica dos sólidos $\left(\mathrm{m}^{2} / \mathrm{s}\right)$} \\
\hline $\mathrm{X}_{\mathrm{w}}-$ Fração mássica da água (adimensional) & \\
\hline X - Fração mássica dos sólidos (adimensional) & \\
\hline
\end{tabular}

\subsection{4 - Condutividade térmica}

A condutividade térmica, em triplicata, das polpas do bacuri foi determinada pela Equação 5 [19], que relaciona os valores experimentais de massa específica, calor específico e difusividade térmica: 
$k=\alpha p C_{p}$

Onde:

$\mathrm{k}$ - Condutividade térmica $\left(\mathrm{W} / \mathrm{m}^{\circ} \mathrm{C}\right)$

$\alpha$ - Difusividade térmica $\left(\mathrm{m}^{2} / \mathrm{s}\right)$

$\rho$ - Massa específica $\left(\mathrm{kg} / \mathrm{m}^{3}\right)$

$\mathrm{C}_{\mathrm{p}}$ - Calor específico $\left(\mathrm{kJ} / \mathrm{kg}^{\circ} \mathrm{C}\right)$

Também foram calculados valores teóricos de condutividade térmica utilizando-se equações proposta em literatura, apresentadas na Tabela 4 , que foram selecionadas por serem função de uma medida simples como a umidade.

TABELA 4 - Equações para cálculo da condutividade térmica em alimentos

\begin{tabular}{lc}
\hline Equação & Referência \\
\hline $\mathrm{k}=0,140+0,42 \mathrm{X}_{\mathrm{w}}$ & {$[20]$} \\
$\mathrm{k}=0,53978+0,00418 \times \mathrm{ST}$ & {$[21]$} \\
$\mathrm{k}=0,901-0,967$ exp & \\
\hline $\mathrm{k}-$ - Condutividade térmica experimental $\left(\mathrm{W} / \mathrm{m}^{\circ} \mathrm{C}\right)$ & \\
$\mathrm{M}-$ Umidade (\%) & \\
$\mathrm{X}_{\mathrm{w}}-$ Umidade (adimensional) \\
$\mathrm{ST}$ - Sólidos totais (\%)
\end{tabular}

\section{3 - Análise estatística}

$\mathrm{Na}$ análise estatística dos dados usou-se o programa ASSISTAT, versão 6.7 [23]. Os dados de calor específico, difusividade térmica e condutividade térmica foram avaliados utilizando-se o delineamento inteiramente casualizado, com comparação entre médias pelo teste de Tukey. Os dados de massa específica, foram avaliados segundo o esquema fatorial 7x2x6 ( 7 concentrações, 2 temperaturas e 6 repetições).

\section{3 - RESULTADOS E DISCUSSÃO}

\section{1 - Determinação das propriedades termofísicas}

\subsection{1 - Calor específico}

De acordo com os dados apresentados na Tabela 5, nota-se que o calor específico das amostras diminui com o aumento da concentração, com o menor valor determinado para as amostras a $20^{\circ} \mathrm{Brix}$ e o maior na amostra a $5^{\circ} \mathrm{Brix}$, refletindo o maior valor dessa propriedade para amostra com maior fração de água. Esta informação, que demonstra a influência da concentração sobre o calor específico, indica o volume de investimentos necessários no projeto de instalações de processamento destinadas a trocas de calor, uma vez que parte dos custos se relacionam às dimensões do equipamento, que por sua vez determinam a quantidade de energia a ser adicionada ou removida nos processos de aquecimento e resfriamento [24].

Na concentração $5^{\circ}$ Brix verifica-se uma diferença significativa com relação às demais concentrações. Para as concentrações de 7,5 a $17,5^{\circ} \mathrm{Brix}$, não há diferença estatística, embora os valores decresçam de forma consistente. Para a polpa a $20^{\circ}$ Brix manifesta-se diferença estatisticamente significativa em relação às polpas a 5,0 e a $7,5^{\circ}$ Brix. Outros pesquisadores reportam valores de calor específico para derivados de frutas na mesma faixa da obtida para o bacuri, como $3,346 \mathrm{~kJ} / \mathrm{kg}^{\circ} \mathrm{C}$ para o açaí [21], 3,32 kJ/ $\mathrm{kg}^{\circ} \mathrm{C}$ para o suco de tamarindo [10] e 2,962 $\mathrm{kJ} / \mathrm{kg}^{\circ} \mathrm{C}$ para polpa de banana [25].

TABELA 5 - Valores médios, calculados de seis repetições, do calor específico da polpa de bacuri em função da concentração

\begin{tabular}{lc}
\hline Concentração ('Brix) & Calor específico $\left(\mathbf{k J} / \mathbf{k g}^{\circ} \mathbf{C}\right)$ \\
\hline 5,0 & $3,616^{\mathrm{a}}$ \\
7,5 & $3,239^{\mathrm{b}}$ \\
10,0 & $3,147^{\mathrm{bc}}$ \\
12,5 & $3,123^{\mathrm{bc}}$ \\
15,0 & $3,105^{\mathrm{bc}}$ \\
17,5 & $3,093^{\mathrm{bc}}$ \\
20,0 & $2,986^{\mathrm{c}}$ \\
\hline
\end{tabular}

DMS $=0,1703 ; \mathrm{MG}=3,187 \mathrm{~kJ} / \mathrm{kg}^{\circ} \mathrm{C} ; \mathrm{CV} \%=2,96$

DMS - Desvio mínimo significativo; MG - Média geral; CV - Coeficiente de variação Obs.: Médias seguidas pela mesma letra minúscula não diferem estatisticamente pelo teste de Tukey, a $5 \%$ de probabilidade

De acordo com os resultados apresentados na Tabela 6 , onde são vistos os valores de calor específico para as amostras em diferentes concentrações, calculados por equações propostas por outros autores, constata-se que a equação proposta por MANOHAR et al. [10] para suco de tamarindo resultou nos menores erros percentuais quando se compara os resultados calculados com os resultados obtidos experimentalmente. A utilização das demais equações produziu os menores erros na polpa a $5^{\circ} \mathrm{Brix}$, em valores próximos de $11,5 \%$, enquanto nas amostras a partir de $7,5^{\circ}$ Brix os erros se mantiveram em torno de $20 \%$.

Na Tabela 7, são apresentados os resultados das regressões do tipo linear, quadrática e exponencial ajustados aos dados experimentais do calor específico, em função da concentração, para a polpa de bacuri. Vê-se que a equação quadrática resultou no melhor ajuste, com coeficiente de determinação superior a $0,86 \%$, sendo recomendada para predizer o calor específico da polpa de bacuri utilizando o valor da concentração entre 5 e $20^{\circ} \mathrm{Brix}$. 
TABELA 6 - Valores de calor específico calculados através de equações existentes na literatura

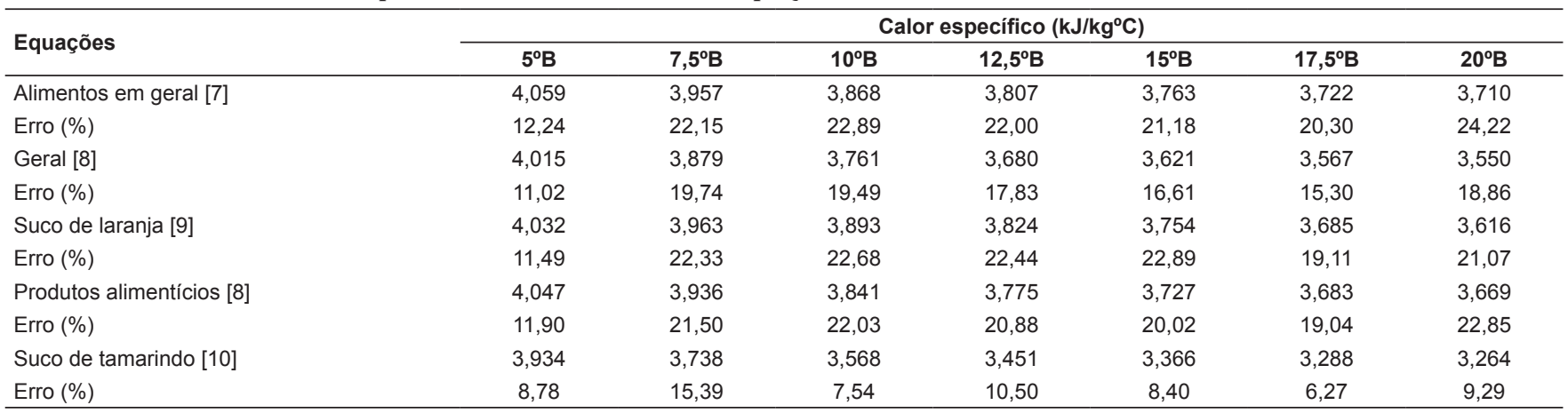

TABELA 7 - Equações propostas para cálculo do calor específico da polpa de bacuri em função da concentração

\begin{tabular}{lcccc}
\hline Equação & $\mathbf{a}$ & $\mathbf{b}$ & $\mathbf{c}$ & $\mathbf{R}^{2}$ \\
\hline$C_{p}=\mathrm{a}+\mathrm{bB}$ & 3,5884 & $-0,0318$ & - & 0,7109 \\
$C_{p}=\mathrm{a}+\mathrm{bB}+\mathrm{cB}^{2}$ & 4,0254 & $-0,1158$ & 0,0034 & 0,8602 \\
$C_{p}=\mathrm{ae}^{\mathrm{bB}}$ & 3,6145 & $-0,0102$ & - & 0,7251 \\
\hline$C_{p}-\left(\mathrm{kJ} / \mathrm{kg}^{\circ} \mathrm{C}\right) ; \mathrm{B}-\left({ }^{\circ} \mathrm{Brix}\right) ; \mathrm{a}, \mathrm{b}$ e c - constantes & &
\end{tabular}

TABELA 8 - Valores médios, calculados de seis repetições, da massa específica da polpa de bacuri, em função da concentração e temperatura

\begin{tabular}{lcc}
\hline \multirow{2}{*}{ Concentração ('Brix) } & \multicolumn{2}{c}{ Massa específica $\left(\mathbf{k g} / \mathbf{m}^{3}\right)$} \\
\cline { 2 - 3 } & $\mathbf{2 5}{ }^{\circ} \mathbf{C}$ & $\mathbf{3 0}$ \\
\hline 5 & $1.009,95^{\mathrm{fA}}$ & $1.008,40^{\mathrm{gB}}$ \\
7,5 & $1.021,76^{\mathrm{eA}}$ & $1.012,35^{\mathrm{fB}}$ \\
10 & $1.034,10^{\mathrm{dA}}$ & $1.026,28^{\mathrm{eB}}$ \\
12,5 & $1.038,31^{\mathrm{CA}}$ & $1.033,01^{\mathrm{dB}}$ \\
15 & $1.040,61^{\mathrm{bA}}$ & $1.038,46^{\mathrm{CB}}$ \\
17,5 & $1.047,05^{\mathrm{aA}}$ & $1.041,85^{\mathrm{bB}}$ \\
20 & $1.048,36^{\mathrm{aA}}$ & $1.044,44^{\mathrm{aB}}$ \\
\hline
\end{tabular}

DMS para colunas $=0,89 ;$ DMS para linhas $=1,36 ; \mathrm{MG}=1.031,77 \mathrm{~kg} / \mathrm{m}^{3} ; \mathrm{CV} \%=0,07$ DMS - Desvio mínimo significativo; MG - Média geral; CV - Coeficiente de variação Obs.: Médias seguidas pela mesma letra minúscula nas colunas e maiúscula nas linhas, não diferem estatisticamente pelo teste de Tukey, a $5 \%$ de probabilidade

\subsection{2 - Massa específica}

Têm-se, na Tabela 8, os valores médios de massa específica da polpa de bacuri, determinados nas concentrações de 5 a $20^{\circ}$ Brix e temperaturas de 25 e $30^{\circ} \mathrm{C}$. Esta propriedade é muito utilizada, em especial, para determinar a concentração de compostos que se encontram na solução, os sólidos presentes no produto e para identificar os diversos materiais [26]. O aumento da massa específica com os aumentos da concentração e sua redução com os aumentos de temperatura, são confirmados nas medições e demonstrados estatisticamente.

Na temperatura de $25^{\circ} \mathrm{C}$ a massa específica aumentou em $3,8 \%$ entre 5 e $20^{\circ} \mathrm{Brix}$, enquanto na temperatura de $30^{\circ} \mathrm{C}$ aumentou 3,6\% entre as mesmas concentrações. Com relação às duas temperaturas, a massa específica diminuiu com o aumento da temperatura em todas as concentrações e essas reduções variaram entre 0,15\% e 0,92\%. Reduções da massa específica com o aumento da temperatura foram observadas para suco de maçã clarificado [27], suco de goiaba [28], suco de laranja [29] e polpa de cupuaçu [13].

Na Tabela 9, tem-se os valores de massa específica calculados segundo equações proposta em literatura. Pela equação de ALVARADO \& ROMERO [12], houve um aumento nos erros percentuais conforme a concentração foi aumentada a partir da concentração de $12,5^{\circ}$ Brix. Isto

TABELA 9 - Valores de massa específica, calculados através de equações existentes na literatura

\begin{tabular}{|c|c|c|c|c|c|c|}
\hline \multirow{3}{*}{ Concentração ( ${ }^{\circ}$ Brix) } & \multicolumn{6}{|c|}{ Massa específica $\left(\mathrm{kg} / \mathrm{m}^{3}\right)$} \\
\hline & \multicolumn{2}{|c|}{ Sucos e polpas de frutas em geral [12] } & \multicolumn{2}{|c|}{ Polpa de cupuaçu [13] } & \multicolumn{2}{|c|}{ Polpa de açaí [14] } \\
\hline & $25^{\circ} \mathrm{C}$ & $30^{\circ} \mathrm{C}$ & $25^{\circ} \mathrm{C}$ & $30^{\circ} \mathrm{C}$ & $25^{\circ} \mathrm{C}$ & $30^{\circ} \mathrm{C}$ \\
\hline 5 & $1,016,49$ & $1,015,07$ & $1,029,42$ & $1,027,28$ & $1,015,52$ & $1,013,59$ \\
\hline Erro (\%) & 0,65 & 0,51 & 1,93 & 1,72 & 0,55 & 0,36 \\
\hline 7,5 & $1,028,02$ & $1,026,60$ & $1,029,42$ & $1,027,28$ & $1,017,39$ & $1,015,46$ \\
\hline Erro (\%) & 0,61 & 0,47 & 0,75 & 0,54 & 0,43 & 0,62 \\
\hline 10 & $1,039,54$ & $1,038,12$ & $1,029,42$ & $1,027,28$ & $1,019,00$ & $1,017,07$ \\
\hline Erro (\%) & 0,53 & 0,39 & 0,45 & 0,66 & 1,46 & 1,65 \\
\hline 12,5 & $1,051,07$ & $1,049,65$ & $1,029,42$ & $1,027,28$ & $1,020,11$ & $1,018,18$ \\
\hline Erro (\%) & 1,23 & 1,09 & 0,86 & 1,06 & 1,75 & 1,94 \\
\hline 15 & $1,062,59$ & $1,061,17$ & $1,029,42$ & $1,027,28$ & $1,020,91$ & $1,018,98$ \\
\hline Erro (\%) & 2,11 & 1,98 & 1,07 & 1,28 & 1,89 & 2,08 \\
\hline 17,5 & $1,074,12$ & $1,072,70$ & $1,029,42$ & $1,027,28$ & $1,021,66$ & $1,019,73$ \\
\hline Erro (\%) & 2,59 & 2,45 & 1,68 & 1,89 & 2,42 & 2,61 \\
\hline 20 & $1,085,64$ & $1,084,22$ & $1,029,42$ & $1,027,28$ & $1,021,89$ & $1,019,96$ \\
\hline Erro (\%) & 3,56 & 3,42 & 1,81 & 2,01 & 2,52 & 2,71 \\
\hline
\end{tabular}


também foi observado no uso da equação proposta por PEREIRA et al. [14], cujos erros percentuais, no entanto, aumentaram já a partir das polpas a $10^{\circ} \mathrm{Brix}$. Na utilização da equação proposta por ARAÚJO et al. [13] os erros tenderam a aumentar nas amostras a partir de $12,5^{\circ} \mathrm{Brix}$. De maneira geral, verifica-se que todas as equações se adequaram aos dados experimentais de forma conveniente, com o maior dos erros não atingindo $4 \%$.

Os resultados das equações de ajuste do tipo linear e quadrático aos valores experimentais de massa específica em função da concentração, para as temperaturas de 25 e $30^{\circ} \mathrm{C}$, se encontram na Tabela 10 . Os ajustes lineares resultaram em valores razoáveis, com $\mathrm{R}^{2}$ acima de 0,9, porém, nos ajustes por equação quadrática verificam-se coeficientes de determinação superiores a 0,98.

Desta forma, estas equações podem ser utilizadas na estimativa da massa específica da polpa de bacuri utilizando o teor de sólidos solúveis totais entre 5 e $20^{\circ}$ Brix. ARAÚJO et al. [13], utilizaram para predição de massa específica de amostras de polpa de cupuaçu integral uma equação cúbica, obtendo valores de $\mathrm{R}^{2}$ acima de 0,99 .

TABELA 10 - Equações propostas para o cálculo da massa específica da polpa de bacuri, em função da concentração

\begin{tabular}{lccccc}
\hline Temperatura $\left({ }^{\circ} \mathrm{C}\right)$ & Equação & $\mathbf{a}$ & $\mathbf{b}$ & $\mathbf{c}$ & $\mathbf{R}^{2}$ \\
\hline \multirow{2}{*}{25} & $\rho=\mathrm{a}+\mathrm{bB}$ & $1.003,53$ & 2,4617 & - & 0,9092 \\
& $\rho=a+b B+\mathrm{cB}^{2}$ & 982,07 & 6,5493 & $-0,1635$ & 0,9844 \\
30 & $\rho=a+b B$ & 997,23 & 2,5615 & & 0,9429 \\
& $\rho=a+b B+\mathrm{cB}^{2}$ & 981,72 & 5,5170 & $-0,1182$ & 0,9806
\end{tabular}

$\rho$ - Massa especifica $\left(\mathrm{kg} / \mathrm{m}^{3}\right)$; B - Concentração ( $\left.{ }^{\circ} \mathrm{Brix}\right) ; \mathrm{a}, \mathrm{b}$ e c - constantes

\subsection{2 - Difusividade térmica}

Observando-se a Tabela 11, onde se apresentam os valores de difusividade térmica para as polpas nas sete concentrações, vê-se que a difusividade decresce com o aumento da concentração. Estatisticamente, os decréscimos se evidenciam de 5 a $12,5^{\circ}$ Brix e entre 12,5 e $20^{\circ}$ Brix. Este comportamento significa, fisicamente, que o aumento da concentração diminui a velocidade com que o calor se propaga ou se difunde através da polpa, a qual é representada pelo valor da difusividade térmica, sendo uma propriedade muito importante para a simulação de processos em que a transferência de calor ocorre em estado transitório [30].

MOURA et al. [31], ao trabalharem com soluções modelo similares a sucos de frutas, também constataram que a difusividade térmica diminuiu com o aumento da concentração, verificando para teores de sólidos totais entre $7,30 \%$ e $15,52 \%$ valores de difusividade térmica entre $1,43 \times 10^{-7} \mathrm{e}$ $0,80 \times 10^{-7} \mathrm{~m}^{2} / \mathrm{s}$, respectivamente. AZOUBEL et al. [32], ao trabalharem com suco de caju nas concentrações de 5,5 a $25^{\circ} \mathrm{Brix}$, igualmente constataram que a difusividade térmica decresce com o aumento dos sólidos solúveis totais das amostras. Os mesmos autores reportam difusividades de
$1,44 \times 10^{-7} \mathrm{~m}^{2} / \mathrm{s}$ e de $1,33 \times 10^{-7} \mathrm{~m}^{2} / \mathrm{s}$ para amostras a $5^{\circ} \mathrm{Brix}$ e a $25^{\circ} \mathrm{Brix}$, respectivamente, inferiores, portanto, aos determinados no presente trabalho para polpas de bacuri nas mesmas concentrações.

A difusividade térmica é uma propriedade importante para a simulação de processos e seu significado físico

TABELA 11 - Valores médios, calculados de três repetições, da difusividade térmica da polpa de bacuri, em função da concentração

\begin{tabular}{lc}
\hline Concentração ${ }^{\circ}$ Brix) & Difusividade térmica $\left(\mathbf{m}^{2} / \mathbf{s}\right)$ \\
\hline 5 & $1,787 \times 10^{-7} \mathrm{a}$ \\
7,5 & $1,763 \times 10^{-7} \mathrm{~b}$ \\
10 & $1,707 \times 10^{-7} \mathrm{c}$ \\
12,5 & $1,673 \times 10^{-7} \mathrm{~d}$ \\
15 & $1,670 \times 10^{-7} \mathrm{~d}$ \\
17,5 & $1,660 \times 10^{-7} \mathrm{~d}$ \\
20 & $1,637 \times 10^{-7} \mathrm{e}$ \\
\hline
\end{tabular}

DMS $=0,022 \times 0^{-7} ; \mathrm{MG}=1,699 \times 10^{-7} \mathrm{~m}^{2} / \mathrm{s} ; \mathrm{CV} \%=0,48$

DMS - Desvio mínimo significativo; MG - Média geral e CV - Coeficiente de variação Obs.: Médias seguidas pela mesma letra não diferem estatisticamente pelo teste de Tukey, a $5 \%$ de probabilidade

Na Tabela 12, apresentam-se os valores da difusividade térmica calculados a partir de modelos citados em literatura, como também os erros em relação às determinações experimentais. Observa-se que a equação para suco de laranja [21] resultou nos menores erros percentuais, com média de $18 \%$ seguida pela equação para alimentos em geral [20], em torno de $20 \%$. A equação para suco de tomate [18], apresentou os maiores erros percentuais, chegando a $49,80 \%$.

TABELA 12 - Valores de difusividade térmica calculados através de equações propostas em literatura

\begin{tabular}{lccc}
\hline \multirow{2}{*}{$\begin{array}{l}\text { Concentração } \\
\text { ('Brix) }\end{array}$} & \multicolumn{3}{c}{ Difusividade térmica $\left(\mathrm{m}^{2} / \mathbf{s}\right)$} \\
\cline { 2 - 4 } & $\begin{array}{c}\text { Alimentos em } \\
\text { geral [16] }\end{array}$ & $\begin{array}{c}\text { Suco de } \\
\text { laranja [17] }\end{array}$ & $\begin{array}{c}\text { Suco de } \\
\text { tomate [18] }\end{array}$ \\
\hline 5 & $1,408 \times 10^{-7}$ & $1,440 \times 10^{-7}$ & $2,677 \times 10^{-7}$ \\
Erro (\%) & 21,21 & 19,42 & 49,80 \\
7,5 & $1,385 \times 10^{-7}$ & $1,416 \times 10^{-7}$ & $2,570 \times 10^{-7}$ \\
Erro (\%) & 21,44 & 19,68 & 45,77 \\
10 & $1,365 \times 10^{-7}$ & $1,395 \times 10^{-7}$ & $2,477 \times 10^{-7}$ \\
Erro (\%) & 20,04 & 18,28 & 45,11 \\
12,5 & $1,351 \times 10^{-7}$ & $1,380 \times 10^{-7}$ & $2,413 \times 10^{-7}$ \\
Erro (\%) & 19,25 & 17,51 & 44,23 \\
15 & $1,341 \times 10^{-7}$ & $1,370 \times 10^{-7}$ & $2,367 \times 10^{-7}$ \\
Erro (\%) & 19,70 & 17,96 & 41,74 \\
17,5 & $1,332 \times 10^{-7}$ & $1,360 \times 10^{-7}$ & $2,325 \times 10^{-7}$ \\
Erro (\%) & 19,76 & 18,07 & 40,06 \\
20 & $1,329 \times 10^{-7}$ & $1,357 \times 10^{-7}$ & $2,311 \times 10^{-7}$ \\
Erro (\%) & 18,81 & 17,10 & 41,17 \\
\hline
\end{tabular}

Na Tabela 13, tem-se os parâmetros das equações do tipo linear, quadrática e exponencial ajustadas aos dados experimentais de difusividade térmica em função da concentração da polpa de bacuri. Pelo critério do coeficiente de determinação constata-se que a equação do tipo quadrática foi a que melhor se ajustou aos dados experimentais, 
seguida pela equação do tipo exponencial, podendo serem utilizadas na estimativa da difusividade térmica da polpa de bacuri utilizando os valores dos sólidos solúveis totais entre 5 e $20^{\circ}$ Brix.

TABELA 13 - Equações propostas para o cálculo da difusividade térmica da polpa de bacuri em função da concentração

\begin{tabular}{lcccc}
\hline Equação & $\mathbf{a}$ & $\mathbf{b}$ & $\mathbf{c}$ & $\mathbf{R}^{2}$ \\
\hline$\alpha-\mathrm{a}+\mathrm{bB}$ & $1,8233 \times 10^{-7}$ & $-0,0099 \times 10^{-7}$ & - & 0,9131 \\
$\alpha=\mathrm{a}+\mathrm{bB}+\mathrm{cB}^{2}$ & $1,8976 \times 10^{-7}$ & $-0,0240 \times 10^{-7}$ & $0,0006 \times 10^{-7}$ & 0,9690 \\
$\alpha=\mathrm{ae}^{\mathrm{bB}}$ & $1,8280 \times 10^{-7}$ & $-0,0059$ & - & 0,9187 \\
\hline$\alpha=\left(\mathrm{m}^{2} / \mathrm{s}\right) ; \mathrm{B}=\left({ }^{\circ} \mathrm{Brix}\right) ; \mathrm{a}, \mathrm{b}$ e c $=$ constantes & &
\end{tabular}

\subsection{3 - Condutividade térmica}

Observa-se, na Tabela 14, os valores de condutividade térmica para as polpas de bacuri. Assim como a difusividade, a condutividade térmica também decresce com o aumento da concentração, significando que a capacidade para conduzir calor da polpa de bacuri diminui com a concentração. Comportamento idêntico também foi observado para sucos de goiaba nas concentrações de 10 a $40^{\circ} \mathrm{Brix}$, e temperatura de $30^{\circ} \mathrm{C}$ [28].

Para as concentrações de 5 a $12,5^{\circ}$ Brix observa-se diferença significativa entre os valores de condutividade, assim como entre 12,5 e $20^{\circ} \mathrm{Brix}$, demonstrando comportamento semelhante ao da difusividade térmica. Para suco de caju na temperatura de $30^{\circ} \mathrm{C}$ e concentração de $5^{\circ} \mathrm{Brix}$, consta na literatura [32] valores de difusividade térmica de $0,58 \mathrm{~W} / \mathrm{m}^{\circ} \mathrm{C}$ e, para a concentração de $20^{\circ} \mathrm{Brix}$ valor de $0,48 \mathrm{~W} / \mathrm{m}^{\circ} \mathrm{C}$, sendo inferiores aos da polpa de bacuri.

TABELA 14 - Valores médios, calculados de três repetições, da condutividade térmica da polpa de bacuri em função da concentração

\begin{tabular}{lc}
\hline Concentração $\left({ }^{\circ}\right.$ Brix $)$ & Condutividade térmica $\left(\mathbf{W} / \mathrm{m}^{\circ} \mathrm{C}\right)$ \\
\hline 5,0 & $0,64 \mathrm{a}$ \\
7,5 & $0,58 \mathrm{~b}$ \\
10 & $0,55 \mathrm{c}$ \\
12,5 & $0,54 \mathrm{~d}$ \\
15 & $0,54 \mathrm{~d}$ \\
17,5 & $0,53 \mathrm{~d}$ \\
20 & $0,50 \mathrm{e}$ \\
\hline
\end{tabular}

DMS $=0,012 ; \mathrm{MG}=0,554 \mathrm{~W} / \mathrm{m}^{\circ} \mathrm{C} ; \mathrm{CV}(\%)=0,79$

DMS - Desvio mínimo significativo; MG - Média geral e CV - Coeficiente de variação Obs.: Médias seguidas pela mesma letra não diferem estatisticamente pelo teste de Tukey, a $5 \%$ de probabilidade

Analisando-se a Tabela 15, onde se tem valores de condutividade térmica calculados por meio de equações propostas em literatura, observa-se que para a equação proposta para sucos de frutas [20], o menor erro percentual foi encontrado para a amostra na concentração de $20^{\circ} \mathrm{Brix}$ com o percentual de $3,96 \%$, enquanto o maior, perfazendo $12,28 \%$, foi medido na concentração de $5^{\circ}$ Brix.

Na equação proposta para polpa de açaí [21], o menor erro percentual encontrado foi de $0,25 \%$ na concentração de $7,5^{\circ}$ Brix e o maior $23,84 \%$ para a amostra a $20^{\circ}$ Brix. Para a equação proposta para banana [22], o menor valor percentual foi obtido para a polpa a $5^{\circ} \mathrm{Brix}$, com o percentual de $0,24 \%$ e o maior para a polpa a $12,5^{\circ}$ Brix $(12,30 \%)$.

TABELA 15 - Valores de condutividade térmica calculados através de equações propostas em literatura

\begin{tabular}{lccc}
\hline \multirow{2}{*}{$\begin{array}{l}\text { Concentração } \\
\text { ('Brix) }\end{array}$} & \multicolumn{3}{c}{ Condutividade térmica $\left(\mathbf{W} / \mathbf{m}^{\circ} \mathrm{C}\right)$} \\
\cline { 2 - 4 } & $\begin{array}{c}\text { Sucos de frutas } \\
{[\mathbf{2 0 ]}}\end{array}$ & $\begin{array}{c}\text { Polpa de açaí } \\
\text { [21] }\end{array}$ & Banana [22] \\
\hline 5 & 0,5386 & 0,5611 & 0,6449 \\
Erro (\%) & 12,28 & 12,78 & 0,24 \\
7,5 & 0,5215 & 0,5781 & 0,6299 \\
Erro (\%) & 9,57 & 0,25 & 9,23 \\
10 & 0,5067 & 0,5928 & 0,6162 \\
Erro (\%) & 8,43 & 7,13 & 11,36 \\
12,5 & 0,4965 & 0,6030 & 0,6064 \\
Erro (\%) & 8,06 & 11,67 & 12,30 \\
15 & 0,4892 & 0,6103 & 0,5990 \\
Erro (\%) & 8,43 & 13,72 & 11,61 \\
17,5 & 0,4823 & 0,6171 & 0,5921 \\
Erro (\%) & 9,00 & 16,43 & 11,72 \\
20 & 0,4802 & 0,6192 & 0,5899 \\
Erro (\%) & 3,96 & 23,84 & 17,98 \\
\hline
\end{tabular}

Na Tabela 16, apresentam-se os parâmetros das regressões dos tipos linear, quadrática e exponencial, ajustados aos dados experimentais da condutividade térmica em função da concentração para a polpa de bacuri. A equação exponencial foi a que melhor se ajustou, com coeficiente de determinação superior a $0,95 \%$, podendo ser utilizada na predição da condutividade térmica da polpa de bacuri utilizando os valores da concentração entre 5 e $20^{\circ}$ Brix.

TABELA 16 - Equações propostas para o cálculo da condutividade térmica da polpa de bacuri em função da concentração

\begin{tabular}{|c|c|c|c|c|}
\hline Equação & a & b & c & $\mathbf{R}^{2}$ \\
\hline$k=a+b B$ & $0,65065 \times 10^{-5}$ & $-0,00771$ & - & 0,8349 \\
\hline$k=a+b B+c B^{2}$ & $0,72237 \times 10^{-5}$ & $-0,02137$ & - & 0,9126 \\
\hline$k=a+b e^{(-B / C)}$ & $0,51261 \times 10^{-5}$ & 0,12702 & 4,49 & 0,952 \\
\hline
\end{tabular}

\section{4 - CONCLUSÕES}

As conclusões que se seguem, de interesse no planejamento de investimentos em unidades de produção de polpa de bacuri como produto processado, com informações sobre demanda por resfriamento e aquecimento nos equipamentos e os gastos energéticos envolvidos, podem ser descritas:

- O calor específico diminuiu com a concentração e a dependência com essa variável pode ser representada 
por uma relação do tipo quadrática. O valor médio do calor específico entre todas as concentrações foi de $3,19 \mathrm{~kJ} / \mathrm{kg}^{\circ} \mathrm{C}$;

- A concentração de 5 a $20^{\circ}$ Brix acarretou aumento na massa específica das polpas em cerca de 3,7\%;

- A difusividade e a condutividade térmica diminuíram com o aumento da concentração. A difusividade em função da concentração foi bem ajustada por uma equação do tipo quadrática e a condutividade ajustouse bem por uma equação do tipo exponencial. O valor médio de difusividade atingiu $1,699 \times 10^{-7} \mathrm{~m}^{2} / \mathrm{s}$ e o valor médio de condutividade situou-se em $0,55 \mathrm{~W} / \mathrm{m}^{\circ} \mathrm{C}$.

\section{5 - REFERÊNCIAS BIBLIOGRÁFICAS}

[1] OLIVEIRA JR., M.E.; MANICA, I. Mercado de frutíferas: principais países e quantidades de frutas produzidas no mundo. Disponível em: http://www. todafruta.com.br. Acesso em: $1^{\circ}$ dez. 2005.

[2] SOUZA, V.A.B.; ARAÚJO, E.C.E.; VASCOMCELOS, L.F.L.; LIMA, P.S.C. Variabilidade das características físicas e químicas de frutos de germoplasma de bacuri da região meio-norte do Brasil. Revista Brasileira de Fruticultura, v. 23, n. 3, p. 677-683, 2001.

[3] FERREIRA, F.R.; FERREIRA, S.A.N.; CARVALHO, J.D.U. Espécies frutíferas pouco exploradas, com potencial econômico e social para o Brasil. Revista Brasileira de Fruticultura, v. 9, n. 2, p. 11-22, 1987.

[4] OLIVEIRA, F.C.; EUGÊNIO, C.E.A.; VASCONCELOS, L.F.L.; SOARES E.B. Métodos para acelerar a germinação de sementes de bacuri (Platonia insignis Mart.). Revista Brasileira de Fruticultura, v. 24, n. 1, p. 151-154, 2002.

[5] VILlACHICA, H.; CARVALHO, J.E.U.; MÜLLER, C.H.; DIAZ, S.C.; ALMANZA M. Frutales y hortalizas promissorios de la Amazonia. Lima: TCA-SPT Publicaciones, 1996.

[6] SILVA, M.; MATA, M.E.R.M.C.; DUARTE, M.E.M.; PEDROZA, J.P.; NASCIMENTO, J.P.T. Resfriamento e propriedades termofísicas do cajá (Spondias lutea L.). Revista Brasileira de Produtos Agroindustriais, Campina Grande, v. 4, n. 2, p. 175-185, 2002.

[7] DICKERSON, R.W. Thermal properties of foods the freezing preservation of foods. $4^{\text {th }}$ ed. Westpoint: The AVI Publishing Company, v. 2, 1968.

[8] CHOI, Y.; OKOS, M.R. Effects of temperature and composition on the thermal properties of foods. In: LEMAUGUER, M.; JELEN, M. Food engineering and process applications: transport phenomena. London: Elsevier Applied Science Publishers, 1986. v. 1, cap. 6, p. 93-101.

[9] VIEIRA, J.A.G. Propriedades termofísicas e convecção no escoamento laminar de suco de laranja em tubos. Campinas, 1996, 87 p. Dissertação (mestrado em Engenharia de Alimentos), Faculdade de Engenharia de Alimentos, Universidade Estadual de Campinas (Unicamp).

[10] MANOHAR, B.; RAMAKRISHNA, P; UDAYASANKAR, $\mathrm{K}$. Some physical properties of tamarind (Tamarindus indica L.) juice concentrates. Journal of Food Engineering, v. 13, n. 4, p. 241-258, 1991.
[11] HIMMELBLAU, D. M. Engenharia química: princípios e cálculos. Rio de Janeiro: Prentice-Hall, 1998. 592 p.

[12] ALVARADO, J.D.; ROMERO, C.H. Physical properties of fruits: I - Density and viscosity of juices as functions of soluble solids and content and temperature. Latin American Applied Research, v. 19, n. 15, p. 15-21, 1989.

[13] ARAÚJO, J.L.; QUEIROZ, A.J.M.; FIGUEIRÊDO, R.M.F. Massa específica de polpa de cupuaçu (Theobroma grandiflorum Schum.) sob diferentes temperaturas. Revista Brasileira de Produtos Agroindustriais, v. 4, n. 2, p. 127-134, 2002.

[14] PEREIRA, E.A.; QUEIROZ, A.J.M.; FIGUEIRÊDO, R.M.F. Massa específica de polpa de açaí em função do teor de sólidos totais e da temperatura. Revista Brasileira de Engenharia Agrícola e Ambiental, v. 6, n. 3, p. 526-530, 2002.

[15] DICKERSON, R.W. An apparatus for the measurement of thermal diffusivity of foods. Food Technology, Westport, v. 19, n. 52, p. 880-886, 1965.

[16] SINGH, R.P. Thermal diffusivity in food processing. Food Technology, p. 87-91, 1982.

[17] TELIS-ROMERO, J.; TELIS, V.R.N.; GABAS, A.L.; YAMASHITA, F. Thermophysical properties of Brazilian orange juice as affected by temperature and water content. Journal of Food Engineering, v. 38, n. 1, p. 27-40, 1998.

[18] CHOI, Y.; OKOS, M.R. The thermal properties of tomato juice concentrates. Transactions of the ASAE, London, v. 26, n. 1, p. 305-311, 1983.

[19] SZCZESNIAK, A.S. Physical properties of food: what they are and their relation to other food properties. In: PELEG, M.; BAGLEY, E.B. Physical properties of foods. Westport: AVI, 1983. p. 1-41.

[20] KOLAROV, K.M.; GROMOV, M.A. Universal equation for calculation of thermal conductivity of fruit and vegetable juices and syrups. Khranitelna Promishhlenost, v. 20, n. 10, p. 33, 1973.

[21] PEREIRA, E.A.; QUEIROZ, A.J.M.; FIGUEIRÊDO, R.M.F. Propriedades termofísicas de polpa de açaí (Euterpe oleracea Mart.). Revista Brasileira de Armazenamento, v. 28, n. 1, p. 17-24, 2003.

[22] NJIE, D.N.; RUMSEY, T.R.; SINGH, R.P. Thermal properties of cassava, yam and plantain. Journal of Food Engineering, v. 37, n. 1, p. 63-76, 1998.

[23] SILVA, F.A.S.; AZEVEDO, C.A.V. Versão do programa computacional Assistat para o sistema operacional Windows. Revista Brasileira de Produtos Agroindustriais, Campina Grande, v. 4, n. 1, p. 71-78, 2001.

[24] MOHSENIN, N.N. Thermal properties of food and agricultural materiais. New York: Gordon and Breach Sci. Publishers, 1980. 407 p.

[25] ALVARADO, J.D. Propiedades fisicas de fruta: difusividad y conductividade térmica efectiva de pulpas. Latin American Applied Research, v. 24, n. 1, p. 41-47, 1994.

[26] ALVARADO, J.D. Propiedades mecânicas: densidad y gravedad específica. In: ALVARADO, J.D.; AGUILERA, J.M. Métodos para medir propiedades físicas em industrias de alimentos. Zaragoza: Acribia, 2001. cap. 1, p. 1-28.

[27] CONSTENLA, D.T.; LOZANO, J.E.; CRAPISTE, G.H. Thermophysical properties of clarified apple juice as 
a function of concentration and temperature. Journal of Food Science, v. 54, n. 3, p. 663-668, 1989.

[28] SHAMSUDIN, R.; MOHAMED, I.O.; YAMAN, N.K.M. Thermophysical properties of Thai seedless guava juice as affected by temperature and concentration. Journal of Food Engineering, v. 66, n. 3, p. 395-399, 2005.

[29] RAMOS, A.M.; IBARZ, A. Density of juice and fruit as a function of soluble solids content and temperature. Journal of Food Engineering, v. 35, n. 1, p. 57-63, 1998.

[30] HUBINGER, M.D.; BARONI, A.F. Propiedades térmicas: conductividad y difusividad térmica. In: ALVARADO,
J.D.; AGUILERA, J.M. Métodos para medir propiedades físicas em industrias de alimentos. Zaragoza: Acribia, 2001. Cap. 2, p. 213-236.

[31] MOURA, S.C.S.R.; FRANÇA, V.C.L.; LEAL, A.M.C.B. Propriedades termofísicas de soluções modelo similares a sucos: parte I. Ciência e Tecnologia de Alimentos, v. 23, n. 1, p. 62-68, 2003.

[32] AZOUBEL, P.M.; CIPRIANI, D.C.; EL-AOUAR, A.A.; ANTONIO, G.C.; MURR, F.E. . Effect of concentration on the physical properties of cashew juice. Journal of Food Engineering, v. 66, n. 4, p. 413-417, 2005. 